\title{
A Novel Clustering and Classification based Approaches for Identifying Tumor in MRI Brain Images
}

\author{
R.Jagadeesan \\ Research Scholar, \\ Department of Electrical and Electronics Enigineering, \\ CMJ University, Meghalaya - 793003.
}

\author{
S. N. Sivanandam, PhD. \\ Reader, \\ Phd Scholar, \\ Department of Electrical and Electronics Enigineering
}

\begin{abstract}
In image processing, image segmentation is one of the important tasks to extract information from the images. A variety of segmentation algorithm is developed to satisfy increasing requirement of image segmentation. Fuzzy CMeans is unsupervised method that has been applied for the variety of purposes such as clustering, classification, image segmentation and target recognition. This method can classify the image, which can be represented in various feature spaces by grouping the similar data points in the feature space into clusters. Especially the FCM can be used to obtain the segmentation with the pixel classification where this method allows pixels to be the property of multiple classes with varying degree of membership. This method can produce the flexibility in processing of Magnetic Resonance Image (MRI). In our proposed first, proposing the K-Means with FCM method and color model to improve the existing system. This algorithm is based on maximum measure of the distance function which is found for cluster center detection process using the Mahalanobis concept. The objective of this research is to develop an enhanced k-means and fuzzy c-means for a segmentation of brain magnetic resonance images. Also we are implementing the Fuzzy Membership based function to select the initial centers for the segmentation process. The firefly algorithm is implemented to optimize the Fuzzy Cmeans membership function for better accuracy segmentation process. At the same time the convergence criteria is fixed for the efficient clustering method. On the whole the proposed technique produces more accurate results compare with other techniques.
\end{abstract}

\section{Keywords}

Fuzzy C-Means Method, K-Means with FCM Method, Magnetic Resonance Image (MRI), Fuzzy Membership Based Function, Firefly Algorithm, Convergence Criteria, Mahalanobis Concept, Image Segmentation, Clustering, Optimized Fuzzy Logic.

\section{INTRODUCTION}

Tumor segmentation from MRI data is a significant although time consuming manual task achieved by medical experts. The process of automating this method is difficult because of high diversity in facade of tumor tissue among different patients and, in many cases, resemblance with normal tissue. This study spotlights on the image segmentation, which is one of the key problems in medical image processing. A new medical image segmentation method is projected based on $\mathrm{k}$ means with fuzzy c- means (FCM) algorithm and color model. Initially, exchange the contrast applied MRI into color model then classify the image into the region of interest and background using FCMs algorithm. The method is accomplished of solving incapable accurately contoured lesion objects problem in MRI image by adding the colorbased segmentation operation. The main idea of colorconverted segmentation algorithm with FCMs is to resolve the given MRI image by translating the input gray-level image into a color space image and operating the image labeled by cluster index.

In this effort, an optimized fuzzy logic method for Magnetic Resonance Imaging (MRI) brain images segmentation is implemented. The process is a method based on a modified fuzzy c-means (FCM) clustering algorithm. The FCM algorithm that have a features as spatial information into the membership function is used for clustering, at the same time as a conventional FCM algorithm does not fully exploit the spatial information in the image. The benefits of the algorithm are that it is fewer sensitive to noise than other methods, and it grants up regions more homogeneous than those of other methods. Innovation of this effort is the techniques practical on a normal MRI brain image and MRI brain images with tumor, and examines the area of tumor from segmented images. The significances demonstrate that the method successfully segmented MRI brain images with spatial information, and the segmented MRI normal brain image and MRI brain images with tumor can be examined for identification principle.

On the whole, the proposed technique produces more accurate results when compared with other techniques. The objective of this research is to develop an enhanced k-means and fuzzy c-means for a segmentation of brain magnetic resonance images. Performance of iterative clustering algorithms which converges to numerous local minima depends highly on initial cluster centers. Generally clustering algorithm chooses the initial centers in random manner. In this paper propose a new center initialization algorithm for measuring the initial centers of the proposed clustering algorithms. This algorithm is based on maximum measure of the distance function which is found for cluster center detection process using the Mahalanobis. Also implementing the Fuzzy Membership based function to select the initial centers for the segmentation process. The firefly algorithm is implemented to optimize the Fuzzy Cmeans membership function for better accuracy segmentation process. At the same time the convergence criteria is fixed for the efficient clustering method.

Fully automatic brain tissue classification from magnetic resonance images (MRI) is of great importance for research and clinical learning of much neurological pathology. Comparing the Fuzzy clustering based segmentation methods along with crisp clustering based methods, the previous one have considerable advantages, since they could preserve much more information from the original image. Fuzzy C-Means (FCM) algorithm one among the fuzzy clustering methods, is a well known method used in image segmentation since it has 
robust characteristics for uncertainty and can retain much more information than crisp clustering methods. The conventional FCM functions well on images with low levels of noise. FCM have two disadvantages when used in segmentation of noise-corrupted images. First one is that the FCM do not integrate the information with reference to the spatial context, which makes it sensitive to the noise and other imaging artifacts.

The main contribution of work is that: As you know, numerous studies of brain segmentation have been proposed, many of which can be categorized into two classes, region detection methods and boundary detection methods, and their goals are to obtain the region and boundary of the desired objects. Meanwhile, most existing segmentation methods are usually dedicated only for specific objects. Combined with the existing methods and aimed to get better results, it's useful to take soft segmentation methods into account. In soft segmentation, voxels are classified into different classes with various degrees of uncertainty which are given by functions. The larger the value of function for a specific voxel, the larger the possibility that this voxel belongs to that cluster.

1. The K-means and fuzzy c-means (FCM) clustering algorithm is soft segmentation method, and it has aroused comprehensive attention. There have been many different families of fuzzy clustering algorithms proposed. The convergence criteria are checked at last and the best clustering will be obtained.

2. The fuzzy Membership function can be used to obtain segmentation via fuzzy pixel clustering. The FCM algorithm that incorporates spatial information into the membership function is used for clustering, while a conventional FCM algorithm does not fully utilize the spatial information in the image.

3. The get an optimized cluster results the firefly algorithm is used. The optimal membership function value is obtained by the firefly algorithm.

The remainder of this paper is as follows: The related work is discussed in section2. The proposed techniques are explained in section 3. The section 4 deals with experimental results and discussion. In section 5 the conclusion of the paper is described.

\section{RELATED WORK}

In this section analysing the existing methods. Juraj Horváth et.al [2] exercised fuzzy c-means clustering method as preprocessing technique for essential region growing segmentation method. Basic dissimilarity from other approaches is conservatory of feature space, which grades in better segmentation. It disquiets insist on borders of segment. Border of segment might be easy and could not be uneven. Wankai Deng et.al [3] offered a hybrid mode, based on modified objective function of FCM and spatial information of images. Hsiang-Chuan Liu et.al [4] changed the common covariance matrix with the correlation matrix in the objective function in the FCM-CM algorithm, after that, a new fuzzy clustering method, called the Fuzzy C-Means algorithm based on standard Mahalanobis distance (FCM-SM), is projected. S. Zulaikha Beevi et.al [5] planned an approach that exploits histogram based Fuzzy C-Means clustering algorithm for the segmentation of MRI medical images. The spatial probability of the neighboring pixels is included in the objective function of FCM to enhance the robustness beside noise.

P. Tamije Selvy et.al [8] used different clustering algorithms like K-means, SOM, Hierarchical Clustering and Fuzzy C-
Means Clustering. The known gray-level MR image is transformed to a color space image and clustering algorithms are applied. The location of tumor objects is cut off as of an MR image by using these clustering algorithms. Hoel Le Capitaine et.al [9] relied on a novel well-organized cluster centers initialization and color quantization permitting faster and further accurate convergence such that it is appropriate to segment enormous color images. Indah Soesanti et.al [10] utilized optimized fuzzy logic method based on FCM clustering that integrates the spatial information into the membership function to get better the segmentation results. The major trouble of this algorithm is that it does not take into account the image topology; it is only based on the pixels values. So, it is very perceptive to noise and in homogeneities in the image in addition, it leftovers reliant on the initialization of the cluster centers.

Abdenour Mekhmoukh et.al [11] anticipated Modified Kernelized Fuzzy C-Means algorithm (MKFCM). It emerges motivating to believe the integration of other limits on the pixels spatial arrangement and to merge several classification algorithms working in cooperation. Hari Prasath [12] presents new medical image segmentation technique based on Enhanced fuzzy c- means (EFCM) algorithm and $\mathrm{YCbCr}$ color model. In this they convert the contrast applied MRI into $\mathrm{YCbCr}$ color model then categorize the image into the region of interest and background by means of EFCMs algorithm. Tuhin Utsab Paul et.al [14] proposed a entirely automated two step segmentation process of brain MRI images. First, the scull is exposed from the MRI images by generating a scull mask from the original MRI image. After all, an advanced $\mathrm{K}$ - means algorithm improvised by two level granularity oriented grid based localization process based on standard local deviation is utilized to segment the image into gray matter, white matter and tumor region. At last, the length and breadth of the tumor is measured. From the all above methods there is no optimality results obtained through this the better segmentation can be achieved.

\section{PROPOSED WORK}

The chore in this problem is to automatically detect the presence of tumors in MR images of the brain, and segment the abnormal pixels from the normal pixels. Conventionally, the task has tried to segment the metabolically active 'enhancing' area of the tumor. A number of current methods have focused on additionally segmenting non-enhancing regions, as well as tumors that may only partially enhance or do not enhance at all. Segmentation of brain tumors from magnetic resonance images is a demanding and timeconsuming task. A semi-automated method has been urbanized for brain tumor and edema segmentation that will provide objective, reproducible segmentations that are close to the manual results. In addition, the method segments nonenhancing brain tumor and edema from healthy tissues in magnetic resonance images.

\subsection{K-Means with Fuzzy C-Means (FCM) Clustering Based Segmentation}

The problem of image segmentation is not anything but a classical clustering problem where the range of image gray values is clustered in some preset number of clustered gray values. The procedure follows a straightforward and easy way to label a given data set during a definite number of $\mathrm{k}$ clusters fixed. These centroids must be positioned in a shrewdness way because of divergent location origin divergent outcome, therefore locate them as much as possible far away from each 
other. The most important application is to label k centroids, one for every cluster.

After this step, find every point be in the right location to the given data set and transmit it to the adjacent centroid. If there are no point is waiting, the first step is completed and an early group time done. In this point there is a need to re-calculate ' $k$ ' new centroids of the clusters ensuing from the earlier step. Subsequent to calculate these $\mathrm{k}$ new centroids, a new fastening has to be done among the equivalent data set points and the adjacent new centroid. A reiteration has been generated. As a result of this loop might well detect that the $\mathrm{k}$ centroids modify their position gradually until no supplementary modifies are done. At last, the algorithms want at the minimizing of objective function, in that case a squared error function where the square is calculated of the distance measured between a data point and the cluster centre, which is the pointer of the distance of the ' $n$ ' data points from their own cluster centre. The algorithm runs through the following steps:

1. Position $\mathrm{K}$ points into the space distinguish by the objects that are being clustered. These points minimally signify initial group centroids.

2. Closest centroids are transferring each object in to a group.

3. Later than assigning each object then recalculation of the positions of the $\mathrm{K}$ centroids is done.

4. Awaiting the centroids no longer move repeat Steps 2 and 3. This is done because of create a disconnection of the objects from the groups from which the metric to be minimized can be calculated.

Although it can be established that the method will always come to an end, the k-means algorithm does not basically locate the best possible arrangement, equivalent to the global objective function minimum. The algorithm is also significantly receptive to the initial arbitrarily chosen cluster centers. Hence, to obtain an optimal segmentation cluster arrangement proposes a normal local deviation guided grid based coarse grain localization after the $\mathrm{K}$ - means algorithm segmentation. At this point, foremost compute the local standard deviation of the $\mathrm{k}$ - means segmented image. Then the histogram of each grid credentials intended and based on the local standard deviation and histogram in each grid, the segmentation boundaries in that grid are reevaluated to produce more optimal segmentation. Choosing a large grid dimension facilitates us reduce the effect of noise in segmenting the grid. But, on the other hand, the large grid dimension also disregards the finer anatomic details for instance twists and turns in the boundary of the tumor or overlapping region of gray and white matters in the brain. So, finally to obtain the most optimal segmentation, we once more process the above segmented image using the same concept of standard local deviation guided grid based fine grain localization. After this process the convergence criteria is implemented to obtain the best optimal solution for segmentation with this FCM.

As you know, the segmentation technique based on FCM only depends on the gray of images, and it doesn't take the spatial information of each voxel into account. Consequently it is essential to offer a more holistic segmentation model. In the FCM algorithm, once the variable $c$ is given, the image can be separated into several parts. On this basis, get to advance segmentation using the spatial information. You know that there are some features are comparable in the same region, while between different regions the features are different. In other words, in the same region, the features modify easily; though, in the boundary the changes are intensity relatively. So the segmentation of the boundary is better, the mean gradient of the boundary is bigger. At the same time, the more the region segmented contains the same tissues, the smaller the mean variance of the region will be. With these spatial features, get the primary method. Foremost, during the modernization of gradient, the gradient image can be obtained by using Sobel operator, and then the area and variance of the interesting region are calculated.

Our aim is to get a congested region on base of the interesting region which gets from the FCM. This closed region must convene the goal that the mean variance adds the reciprocal of the mean gradient is to be minimum. Subsequent to proceed by the FCM method, the preliminary region of tumor tissues is attained, and which is used as the initial region. Then obtain a set of the interesting regions through an iterative process on base of the initial region. First time put the value of the initial region, and the background to be zero. Then we use a window to slide through the picture, when the value in the window is the same as that of the initial region, the region that the window enclosed is joined into the initial region. Deduce that the iteration number is $t$, the set of the interesting regions is $j_{p}$ and its closed outline curve is $l_{j} . i$ Represents gray-level, $0 \leq$ $i<L, n_{i}$ is the number of gray-level $i$ in $p_{j}, n_{j}$ is the number of pixels on the outline $l_{j}$. For every pixel $k$, can obtain the gradient of it and choose the gradient by $k g$ through the gradient image. So mean gradient of each outline is

$G_{l_{j}}=\frac{\sum_{k-1}^{n_{j}} g_{k}}{n_{j}}$

$\beta_{l_{j}}=\frac{1}{G_{l_{j}}}$

At the same time, the mean variance of each region $p_{j}$ can be written as follows:

$\begin{aligned} \mu_{p_{j}} & =\frac{\sum_{i=0}^{L-1}\left(i n_{i}\right)}{\sum_{i=0}^{L-1} n_{i}} \\ \sigma_{p_{j}}^{2} & =\frac{\sum_{i=0}^{L-1}\left(i-\mu_{p_{j}}\right)^{2}}{\sum_{i=0}^{L-1} n_{i}}\end{aligned}$

Thus, a new objective function is defined using the reciprocal of mean gradient and variance as follows:

$s_{j}=\beta_{l_{j}}+\sigma_{p_{j}}^{2}$
$s=\arg \min _{j}\left\{s_{j}\right\}$

From this the optimum results will be got of tumor tissues.

\subsubsection{Fuzzy Membership Function}

The FCM algorithm allocates pixels to every category by using fuzzy memberships. Let $X=\left(x_{1}, x_{2}, ., x_{n}\right)$ indicates an image with $n$ pixels to be separated into c clusters, where $x_{i}$ represents multispectral (features) data. The algorithm is an iterative optimization that reduces the cost function using the Kullback-Leibler (KL) criterion defined as follows:

$J=\sum_{j=1}^{n} \sum_{i=1}^{c} u_{i j}^{m}\left\|\left(x_{j}-v_{i}\right) \log _{2} \frac{x_{j}}{v_{i}}\right\|^{2}$ 
Where $u_{i j}$ symbolizes the membership function of pixel $x_{j}$ in the ith cluster, $v_{i}$ is the ith cluster center, and $\mathrm{m}$ is an invariable. The parameter $m$ manages the fuzziness of the resulting partition, and $m$ is fixed in this revise. The cost function is reduced when pixel close to the centroid of their clusters are assigned high membership values, and low membership values are assigned to pixels with data far from the centroid. The membership function symbolizes the probability that a pixel goes to a specific cluster. In the FCM algorithm, the probability is dependent solely on the distance between the pixel and each individual cluster center in the feature domain. The membership functions and cluster centers are reorganized by the following:

$u_{i j}=\frac{1}{\sum_{k=1}^{c}\left(\frac{\left\|x_{j}-v_{i}\right\|}{\left\|x_{j}-v_{k}\right\|}\right)^{2 /(m-1)}}$

$v_{i}=\frac{\sum_{j=1}^{N} u_{i j}^{m} x_{j}}{\sum_{j=1}^{N} u_{i j}^{m}}$

Where $u_{i j} \in[0,1]$.

Preliminary with an initial guess for each cluster center, the FCM converges to a solution for $v_{i}$ representing the local minimum or a load point of the cost function. Convergence can be observed by comparing the changes in the membership function or the cluster center at two successive iteration steps. One of the significant characteristics of an image is that neighboring pixels possess similar feature values, and the probability that they belong to the same cluster is great. This spatial relationship is important in clustering, but it is not utilized in a standard FCM algorithm. To develop the spatial information, a spatial function is defined as.

$$
h_{i j}=\sum_{k \in N B\left(x_{j}\right)} u_{i k}
$$

Where $N B\left(x_{j}\right)$ represents a square window centered on pixel $x_{j}$ in the spatial domain. Just like the membership function, the spatial function $h_{i j}$ symbolizes the probability that pixel $x_{j}$ belongs to $i$ th cluster. The spatial function of a pixel for a cluster is large if the majority of its neighborhood belongs to the same cluster. The spatial function in incorporated into membership function as follows:

$u_{i j}^{\prime}=\frac{u_{i j}^{p} h_{i j}^{q}}{\sum_{k=1}^{c} u_{k j}^{p} h_{k j}^{q}}$

Where $p$ and $q$ are parameters to control the relative importance of both functions. In a homogenous region, the spatial functions strengthen the original membership, and the clustering result remains unchanged. However, for a noisy pixel, this formula reduces the weighting of a noisy cluster by the labels of its neighboring pixels. As a result, misclassified pixels from noisy regions or spurious splashes can easily be approved. The spatial FCM with parameter $p$ and $q$ is denoted $F C M S_{p, q}$.

The clustering is a two-pass process at each of iteration. The first pass is the same as that in standard FCM to calculate the membership function in the spectral domain. In the second pass, the membership information of each pixel is mapped to the spatial domain, and the spatial function is calculated from that. The FCM iteration proceeds with the new membership that is included with the spatial function. The iteration is stopped when the maximum difference between two cluster centers at two successive iterations is less than a threshold.

\section{FIREFLY BASED OPTIMIZED CLUSTER METHOD}

Firefly Algorithm (FA) is a nature-inspired algorithm based on the flashing behaviors of the firefly swarm [18]. The main principle for the sparkle of fireflies is to signal to attract other fireflies. The assumption of FA consists of three rules. (1) all fireflies are unisex thus that one firefly will be fascinated to other fireflies regardless of their sex; (2) an essential and motivating behavior of fireflies is to radiance brighter mainly to attract victim and to share food with others; (3) attractiveness is comparative to their brightness, so each agent firstly travels toward a neighbor that glows brighter. In the FA, the fireflies are randomly dispersed in the search space. The fireflies hold a luminescence excellence, called luciferin, which emanates light proportional to the quality [16]. Every firefly is attracted by the brighter glow of other estimated fireflies. The attractiveness reduces as their distance increases [17]. If there is no brighter one surrounded by the scope of a firefly, it will travel randomly in the search space. In our applications, the decision variables are the three spatial transform parameters as $t_{x}, t_{y}$ and $\theta$. The brightness is associated as the objective function is formulated as Equation in below section. The actions of applying FA to inflexible image registration can be typed into two phases.

\subsection{Distinction of light intensity}

The brightness is correlated to the objective values, hence for a maximization/minimization problem; a firefly with advanced intensity will attract another firefly with higher probability, and vice versa. Suppose that there survives a swarm of $n$ fireflies and $x_{i}$ stands for a solution for a firefly $i$, whereas $f\left(x_{i}\right)$ signifies its corresponding fitness value. Here the brightness $I$ of a firefly is correspondent to the fitness value

$$
I_{i}=f\left(x_{i}\right) 1 \leq i \leq n
$$

\subsection{Movement in the direction of attractive flies}

The attractiveness $\beta$ of the firefly is proportional to the light intensity obtained by the adjacent fireflies [19]. Assume $\beta_{0}$ is the attractiveness with distance $d=0$, hence for two fireflies $i$ and $j$ at locations $x_{i}$ and $x_{j}$, their attractiveness is calculated as

$$
\begin{gathered}
\beta_{d}(i, j)=\beta_{0} \exp \left\{-\gamma d(i, j)^{2}\right\} \\
d(i, j)=\left\|x_{i}-x_{j}\right\|
\end{gathered}
$$

Where $d(i, j)$ denotes the distance between fireflies $i$ and $j, \gamma$ denotes the light amalgamation coefficient. Understand firefly $j$ is brighter than firefly $i$, then firefly i will move to a new position as

$$
x_{i}(t+1)=x_{i}(t)+\beta_{0} \exp \left\{-\gamma d^{2}\right\}\left(x_{j}-x_{i}\right)
$$

The pseudo code of this algorithm is given by: 


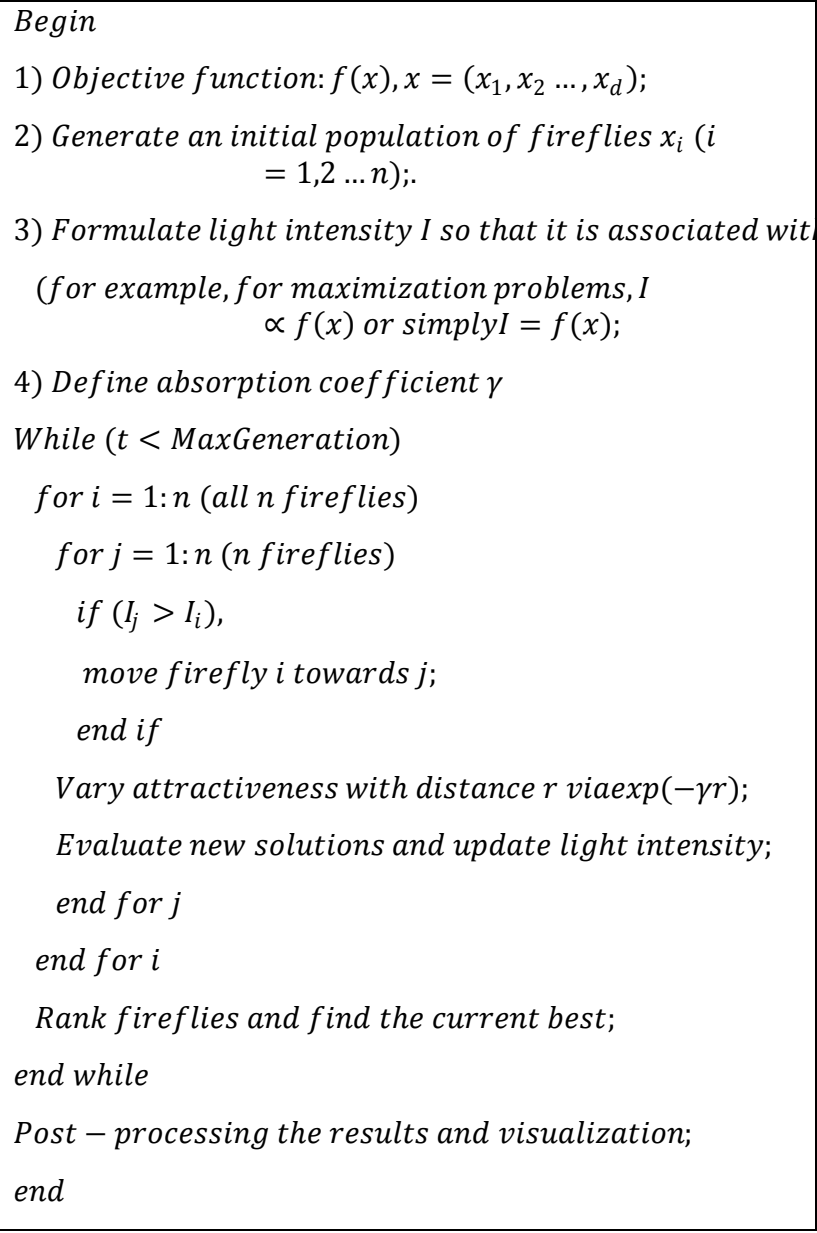

\section{EXPERIMENTAL RESULTS AND DISCUSSION}

In the proposed system, brain tumors were segmented by using different clustering algorithms and optimization algorithm. The results obtained for the clustering algorithms are given in this section. In this the recall rate and the execution time is calculated and compared with the different clustering methods with the support of number of input dataset. Figure 1 represents the recall rate comparison among the three different algorithms. This graph shows the recall rate of three different clustering methods based on two parameters of recall and number of Input images. From the graph can say that, when the number of number of Input images is improved the recall rate also improved in proposed system which is represented as blue line but when the number of number of Input images is improved the recall rate is reduced in two existing systems which are represented as redline and green line. From this graph can say that the recall rate of Firefly based k-Means with FCM is increased which will be the best for the image segmentation.

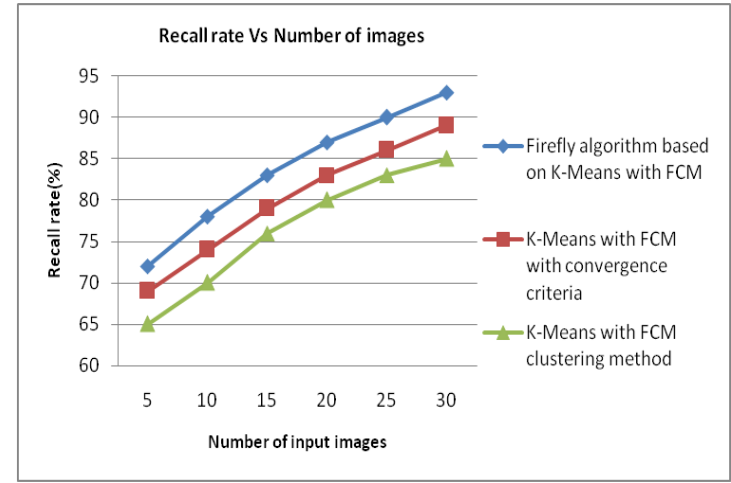

Fig 1: Recall rate comparison

Figure 2 represents the Execution time comparison among the three different algorithms. This graph shows the Execution time of three different clustering methods based on two parameters of Execution time and number of Input images. From the graph can see that, when the number of number of Input images is improved the Execution time is reduced in proposed system which is represented as blue line but when the number of number of Input images is improved the Execution time is increased in two existing systems which are represented as redline and green line. From this graph can say that the Execution time of Firefly based k-Means with FCM is reduced which will be the best for the image segmentation.

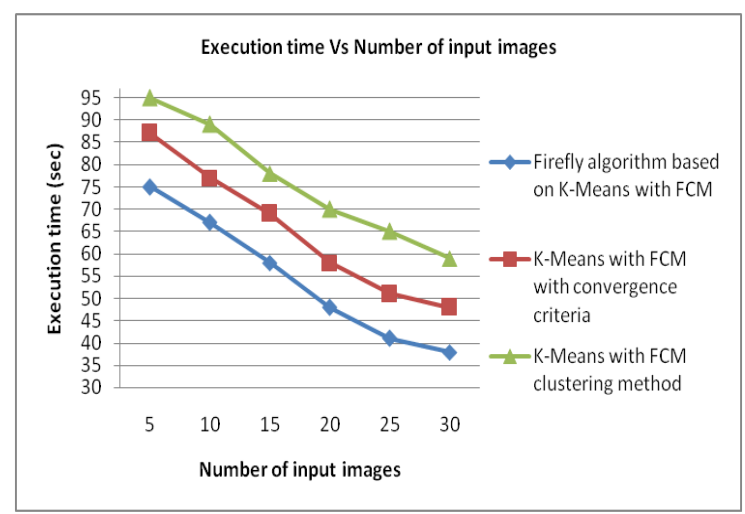

Fig 2: Execution time comparison

\section{CONCLUSION}

In this a realization of tumor cut segmentation method of MRI brain image using k-means with fuzzy C-means clustering technique based on color model. This proposed color-based Segmentation with K-Means with FCM clustering algorithm for tracking tumors in medical images is performed to be very capable for MRI applications. The brain regions related to a tumor or lesion can be exactly separated from the colored image. This proposed method will be able to help pathologists distinguish exactly lesion size and region. With this work implementing the firefly optimization based clustering method to improve the clustering results. The experimental results shows, the proposed system is providing better clustering results than the existing methods. 


\section{REFERENCES}

[1] Dzung L. Pham, Jerry L. Prince, "An Adaptive Fuzzy CMeans Algorithm for Image Segmentation in the Presence of Intensity In homogeneities”, 1998

[2] Juraj Horváth,'Image Segmentation Using Fuzzy CMeans", Department of Cybernetics and Artificial Intelligence, Faculty of Electrical Engineering and Informatics, Technical University of Košice Letná 9, 042 00 Košice.

[3] Wankai Deng, Wei Xiao, Chao Pan, Jianguo Liu,"MRI brain tumor segmentation based on improved fuzzy cmeans method", Proc. of SPIE Vol. 7497, 74972N, 2009

[4] Hsiang-Chuan Liu, Bai-Cheng Jeng, Jeng-Ming Yih, and Yen-Kuei Yu,"Fuzzy C-Means Algorithm Based on Standard Mahalanobis Distances", Proceedings of the 2009 International Symposium on Information Processing (ISIP'09) Huangshan, P. R. China, August 21-23, 2009, pp. 422-427

[5] S. Zulaikha Beevi, M. Mohamed Sathik,"An Effective Approach for Segmentation of MRI Images: Combining Spatial Information with Fuzzy C-Means Clustering", European Journal of Scientific Research ISSN 1450216X Vol.41 No.3 (2010), pp.437-451

[6] Dr.G.Padmavathi, Mr.M.Muthukumar and Mr. Suresh Kumar Thakur,"Non linear Image segmentation using fuzzy c means clustering method with thresholding for underwater images", IJCSI International Journal of Computer Science Issues, Vol. 7, Issue 3, No 9, May 2010

[7] R. Rajeswari, P. Anandhakumar," Segmentation and Identification of Brain Tumor MRI Image with Radix4 FFT Techniques", European Journal of Scientific Research ISSN 1450-216X Vol.52 No.1 (2011), pp.100109

[8] P. Tamije Selvy, V. Palanisamy, T. Purusothaman,"Performance Analysis of Clustering Algorithms in Brain Tumor Detection of MR Images", European Journal of Scientific Research ISSN 1450216X Vol.62 No.3 (2011), pp. 321-330

[9] Hoel Le Capitaine and Carl Frélicot,"A fast fuzzy cmeans algorithm for color image segmentation", Author manuscript, published in "EUSFLAT2011, France (2011)

[10] Indah Soesanti, Adhi Susanto, Thomas Sri Widodo, Maesadji Tjokronagoro,"Optimized Fuzzy Logic Application for MRI Brain Images Segmentation", International Journal of Computer Science \& Information Technology (IJCSIT) Vol 3, No 5, Oct 2011

[11] Abdenour Mekhmoukh, Karim Mokrani and Mohamed Cheriet,"A modified Kernelized Fuzzy C-Means algorithm for noisy images segmentation: Application to MRI images", IJCSI International Journal of Computer Science Issues, Vol. 9, Issue 1, No 1, January 2012

[12] Hari Prasath S.P, G.Kharmega Sundararaj, A.Jayachandran,"Brain Tumor Segmentation of Contras Material Applied MRI Using Enhanced Fuzzy C-Means Clustering", International Journal of Engineering and Innovative Technology (IJEIT) Volume 1, Issue 2, February 2012
[13] R. Harrabi and E. Ben Braiek,"Color Image Segmentation Based on a Modified Fuzzy C-means Technique and Statistical Features", International Journal of Computational Engineering Research / ISSN: 22503005

[14] Tuhin Utsab Paul Samir Kumar Bandhyopadhyay,"Segmentation of Brain Tumor from Brain MRI Images Reintroducing K - Means with advanced Dual Localization Method", International Journal of Engineering Research and Applications (IJERA) ISSN: 2248-9622 www.ijera.com Vol. 2, Issue 3, May-Jun 2012, pp. 226-231

[15] Zhengjian Ding, Jin Sun, and Yang Zhang,'FCM Image Segmentation Algorithm Based on Color Space and Spatial Information" International Journal of Computer and Communication Engineering, Vol. 2, No. 1, January 2013

[16] M.-H. Horng and R.-J. Liou, "Multilevel minimum cross entropy threshold selection based on the firefly algorithm," Expert Systems with Applications, vol. 38, pp. 14805-14811, 2011.

[17] A. H. Gandomi, X.-S. Yang, and A. H. Alavi, "Mixed variable structural optimization using Firefly Algorithm," Computers \&amp; Structures, vol. 89, pp. 2325-2336, 2011.

[18] X.-S. Yang, S. S. Sadat Hosseini, and A. H. Gandomi, "Firefly Algorithm for solving non-convex economic dispatch problems with valve loading effect," Applied Soft Computing, vol. 12, pp. 1180-1186, 2012.

[19] M.-H. Horng, "Vector quantization using the firefly algorithm for image compression," Expert Systems with Applications, vol. 39, pp. 1078-1091, 2012

\section{AUTHORS' PROFILE}

R.Jagadeesan done BE (Electrical Engineering), from Madras University in 1980, completed M.E.(Electrical Engineering), from Bharathiar University in 1988. His research area is Pattern Recognition, Multidimensional system analysis, Image processing.

Dr. S. N. Sivanandam completed his B.E. (Electrical Engineering) in 1964 from Government College of Technology, Coimbatore, and MSc (Engineering) in Power Systems in the year 1966 from PSG College of Technology, Coimbatore. He acquired $\mathrm{PhD}$ in control systems in 1982 from Madras University. He received best teacher award in the year 2001 and Dhakshina Murthy Award for teaching excellence from PSG College of technology. He received the citation for best teaching and technical contribution in the year 2002, Government College of Technology,Coimbatore. His research areas include Modeling and Simulation, Neural Networks, Fuzzy Systems and Genetic Algorithm, Pattern Recognition, Multidimensional system analysis, Linear and Non linear control system, Signal and Image processing, Control System, Power System, Numerical methods, Parallel Computing, Data Mining and Database Security. $\mathrm{He}$ is a member of various professional bodies like IE (India), ISTE, CSI, ACS and SSI. He is a technical advisor for various reputed industries and engineering institutions. 